\title{
Fotometria BVRI da galáxia M49 (NGC 4472): Reduções IRAF e MaxIm DL
}

\author{
Vitor de Oliveira Silva ${ }^{1}$; Vera Aparecida Fernandes Martin²; Paulo Cesar da \\ Rocha Poppe $^{3}$ \\ 1. Bolsista PIBIC/CNPq, Graduando em Bacharelado em Física, Universidade Estadual de Feira de Santana, \\ e-mail: vitorfsa1@gmail.com \\ 2. Orientador, Departamento de Física, Universidade Estadual de Feira de Santana, \\ e-mail:vmartin@uefs.br
}

3. Participante do projeto ou núcleo tal, Departamento de Física, Universidade Estadual de Feira de Santana, e-mail: paulopoppe@uefs.br

PALAVRAS-CHAVE: Fotometria, Redução de dados, Instrumentação

\section{INTRODUÇÃO}

A galáxia Messier 49 (M49, NGC 4472), descoberta por Charles Messier em 1771, é uma galáxia com morfologia elíptica gigante, E2, mas também é classificada como uma galáxia lenticular, S0, (De Vaucouleurs et al. 1991), localizada na constelação de Virgem (ver Figura 1). Foi a primeira galáxia membro do aglomerado de Virgem a ser descoberta e é uma das mais brilhante deste sistema, com magnitude na banda $\mathrm{B}=$ $9,37 \pm 0,06$ (De Vaucouleurs et al. 1991). Representa um objeto extragaláctico (z = $0,003272 \pm 0,00017)$ com velocidade de recessão de $981 \pm 5 \mathrm{~km} / \mathrm{s}$. É classficada com núcleo ativo (AGN - Active Galactic Nuclei), do tipo Seyfert2. Uma classificação LINER (Low-Ionization Nuclear Emission Line Region) também é sugerida. Possu coordenadas equatoriais $\alpha=12^{\mathrm{h}} 29^{\mathrm{m}} 46,7^{\mathrm{s}}$ e $\delta=+8^{\circ} 00^{\prime} 02^{\prime \prime}$.

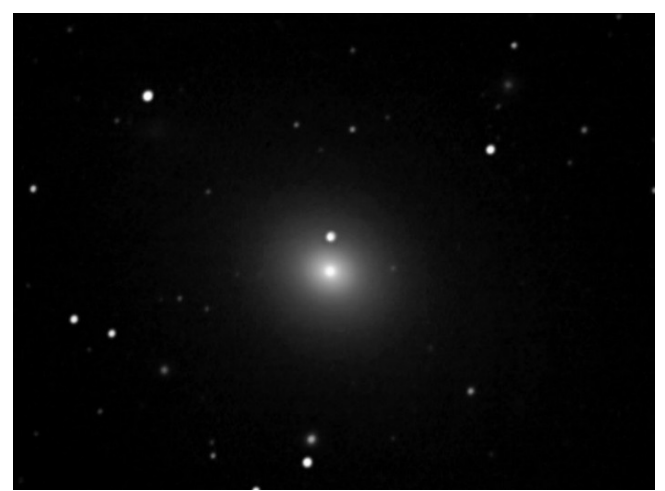

Figura 1: Imagem da galáxia M49 (NGC 4472) na banda R obtida no Observatório Astrônomico Antares - UEFS

Fotometria CCD (BVRI) de objetos extensos representa uma linha de pesquisa que está sendo desenvolvida com o telescópio CDK20 (Corrected Dall-Kirkham Astrograph telescope, de 20 polegadas - 50,8cm de diâmetro), instalado no Observatório Astronômico Antares (OAA). Apesar de ser um objeto bem estudado na literatura com as técnicas de fotometria e espectroscopia (e.g., Foster et al. (2011), Kim (2010), Sanchez-Blazquez et al. (2007), Michard (1985), King (1978)), em diversos comprimentos de onda do espectro eletromagnético, M49 representa um excelente exemplo para iniciar os conceitos de fotometria superficial para os estudantes de Iniciação Científica. 
Em geral, o pacote IRAF (Image Reduction and Analysis Facility), de domínio público, consiste de um sistema de softwares construído para a redução e análise de dados astronômicos, e vem sendo empregado sistematicamente em nossas reduções fotométricas e/ou espectoscópicas. No entanto, apesar da sua ampla validade já assegura pela comunidade astronômica, iremos utilizar um pacote alternativo, MaxIm DL, desenvolvido pela Cyanogen Imaging ${ }^{\circledR}$. Portanto, uma avaliação quanto a performance permitirá avaliar o mesmo para o uso em futuras reduções fotométricas, não apenas de objetos extensos, mas também de estrelas, aglomerados e objetos do Sistema Solar, em particular, asteróides.

\section{MATERIAL E MÉTODOS OU METODOLOGIA (ou equivalente)}

Apesar de ainda não termos uma calculadora de tempo de integração para o CDK20, próximo projeto a ser executado, as observações realizadas para objetos com magnitude aparente semelhante $(\sim 9,0)$, sugere os seguintes tempos de exposição (em minutos) nas respectivas bandas para a supracitada galáxia: Johnson $\mathrm{B}(10), \mathrm{V}(8)$ e Cousins $\mathrm{R}(4)$ e I(3), permitindo obter contagens suficientes para o processo de redução fotométrica. Estrelas padrões espectrofotométricas observadas da lista de Landolt (1983) ou Smith et al. (1991) para os propósitos de calibração, também serão obtidas na mesma noite de observação, porém com tempos de exposições bem inferiores, dependende da magnitude da estrela escolhda, afim de não saturar as mesmas. Usaremos os coeficientes de extinção atmosféricos médios já obtidos para o OAA, apesar de um outro projeto de Iniciação Científica proposto reavaliar os mesmos através de uma outra medologia de estudo. Estes coeficientes servem para corrigir as medidas fotométricas (observações) que são obtidas na presença da atmosfera terrestre. Independente da metodologia de redução escolhida (MaxIm DL ou IRAF), as calibrações instrumentais (bias, dark e flatfield) são necessárias para ambos os casos, em números suficientes para que uma amostragem estatística seja garantida.

\section{RESULTADOS E/OU DISCUSSÃO (ou Análise e discussão dos resultados)}

As observações fotométricas foram obtidas em 22/03/2017 com o telescópio de 0,508m do Observatório Astronômico Antares. O seeing (fenômeno óptico que ocorre nas imagens formadas por telescópios terrestres, na qual a imagem de uma estrela torna-se alargada em decorrência dos movimentos turbulentos da atmosfera) durante as observações foi da ordem de 2,5” (sendo menor do que 1,0” em observatórios com excelentes condições atmosféricas). O brilho do céu, em magnitudes por segundo de arco quadrado, ou seja, o nível de transparência do céu noturno associado aos processos de espalhamento e difusão da luz natural e artificial) para os filtros BVRI foi de 21,75, 20,55, 19,92 e 18,27, respectivamente. O fundo de céu para subtração foi estimado examinando-se a contagem média em anéis concêntricos de diferentes larguras centrado na galáxia com um raio externo estendido ao limite e estimando o nível mais baixo de intensidade. As reduções seguiram os procedimentos padrões usados no pacote livre IRAF (Image Reduction and Analysis Facility) e também no comercial Maxim DL. As estrelas de calibração foram retiradas da lista de Landolt (1993). 


\section{Isofotas elípticas}

As análises isofotais e o modelamento da galáxia foram feitas através das tarefas do pacote STSDAS do IRAF: “ellipse” e ua versão modificado de "bmodel”. A tarefa ellipse ajusta elipses de determinados semi-eixos maiores às imagens. O procedimento utilizado para ajustar elipses fundamenta-se em correções diferenciais que dependem da existência de um gradiente do brilho do objeto com o afastamento da região central. Caso o gradiente seja nulo ou indefinido, o procedimento falha. No entanto, como esta tarefa foi pensada para galáxias normais (não peculiares) da sequência de Hubble (elípticas, lenticulares e espirais), o gradiente é sempre negativo, aproximando-se de zero somente no limite exterior da galáxia, onde esta se confunde com o céu. Para tais objetos, a tarefa foi programada para interromper as tentativas de ajuste de elípses e, adotando o último ajuste bem sucedido, continuar com a mesma elipticidade, posição e centro e do ângulo de posição até os limites impostos pelo usuário ou pelo tamanho da iamgem. A seguinte estratégia foi usada nesse trabalho: as elípses terão seus semi-eixos maiores determinados e o ajuste dos 4 parâmetros geométricos básicos é feito não diretamente, mas investigando-se como a elípse se ajusta aos dados da galáxia. Os parâmetros geométricos são: (i) o semi-eixo maior, a; (ii) o ângulo de posição do semieixo maior com a direção y, aumentado no sentido anti-horário, $\phi$; (iii) a elipticidade, $\varepsilon$, definida em termos dos semi-eixos menor e maior ou em termos da excentricidade e por $\varepsilon=1-\mathrm{b} / \mathrm{a}=1-\left(1-\mathrm{e}^{2}\right)^{1 / 2}$; (iv) a posição do centro da elipse, $\mathrm{x}_{0}$ e $\mathrm{y}_{0}$.

Os semi-eixos maiores (a) são pré-determinados a partir das images nas bandas BVRI. O pacote investiga para determinado valor de (a) qual deveria ser o melhor valor para os demais parâmetros e parte para um estudo de como as intensidades da medida (galáxia) dentro dos valores $\mathrm{x}$ e y se relacionam com o valor da intensidade da elipse isofotal. $\mathrm{O}$ estudo é realizado em termos da anomalia excêntrica utilizando-se uma função do tipo:

$$
I_{i}=I_{0}+\sum_{n=1}^{8} A_{n} \sin \left(n E_{i}\right)+B_{n} \cos \left(n E_{i}\right)
$$

onde $I_{i}$ e $I_{0}$ correspondem à intensidade do ponto $i$ e à intensidade isofotal, respectivamente (magnitude superficial correspondente). $E_{\mathrm{i}}$ é a anomalia excêntrica do ponto i e n é a ordem do harmônico utilizado. Os harmônicos 1 e 2 correspondem à elipse propriamente dita. Relacionando com os respectivos coeficientes $\left(A_{1}, A_{2}, B_{1}\right.$ e $\left.B_{2}\right)$ estão as correções que se deve dar aos parâmetros geométricos para se obter o melhor ajuste (ver o artigo de Jedrzejewski (1987) e "help ellipse option=sysdoc", dentro do IRAF). Estas correções dependem do gradiente acima mencionado do brilho da galáxia em função da distância ao centro da mesma, sendo que este valor entra no denominador das equações para as correções (i.e., precisa de um valor definido e diferente de zero). A forma de se analisar os resultados da análise isofotal consiste em traçar a variação dos mesmos em função da distância ao centro da galáxia. Para tal, podemos empregar os semi-eixos maior e menor, mas existe ainda uma definição um pouco mais elaborada, ou seja, o chamado "raio equivalente", definido, por exemplo, por de Vaucouleurs (1948). O raio equivalente é o raio do círculo que possui a mesma área que a isofota. O IRAF oferece as tarefas “isoplot” e "plotall” para visualizar os perfis de luminosidade e os dos 
parâmetros estruturais gerados pela tarefa ellipse. A Figura 2 mostra o perfil de luminosidade ao longo da média do eixo obdecendo a lei $\mathrm{a}^{1 / 4}$. O parâmetro "a” refere-se ao semi-diâmetro maior da galáxia (em pixels).
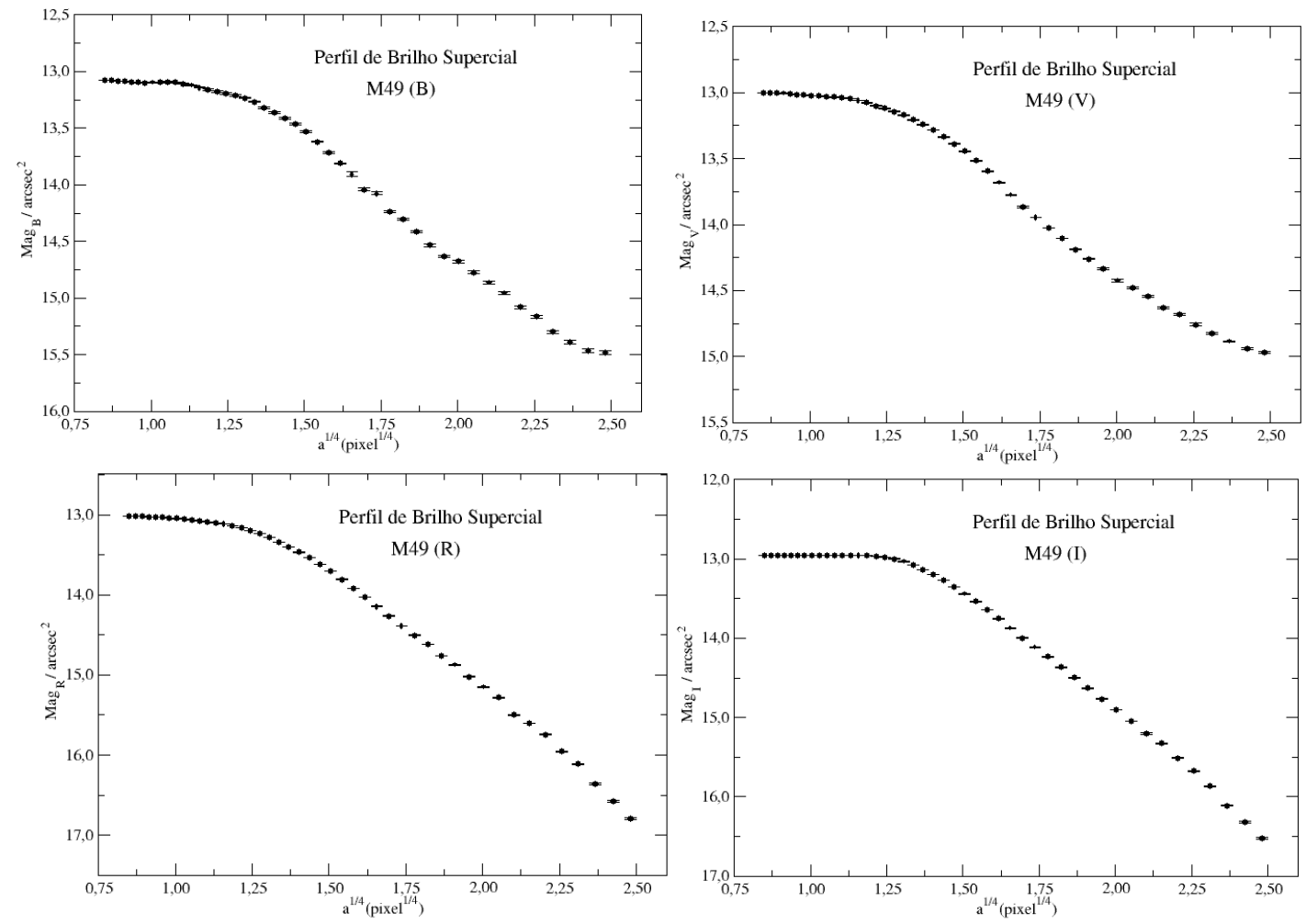

Figura 2. Superfície isofotal para cada galáxia em função de $a^{1 / 4}$. (perfil de de Vaucouleurs)

\section{CONSIDERAÇÕES FINAIS (ou Conclusão)}

O IRAF é uma ampla coleção de softwares para uso geral em redução e análise de dados científicos, desenvolvido e mantido pelo National Optical Astronomy Observatory, em Tucson, Arizona (USA). Trata-se, portanto, do melhor pacote empregado pelo nosso grupo para a redução fotométrica e espectroscópica de dados. Por outro lado, o MaxIm DL oferece facilidades por trabalhar na forma de janelas (diferentemente das linhas de comando do IRAF no Linux), possibilitando uma calibração rápida e robusta. No entanto, não apresenta os recursos disponíveis no IRAF para o tratamento fotométrico para obter as curvas descritas nesse trabalho.

\section{REFERÊNCIAS}

De Vaucouleurs G., De Vaucouleurs A., Corwin JR. H.G., Buta R.J., Paturel G., Fouque P., 1991, RC3, 9

Foster C., Spitler L. R., Romanowsky A.J., (+ 7 co-autores), 2011, MNRAS, 415, 3393

Jedrzejewski, R. I., MNRAS, 226, 747, 1987

King I., 1978, ApJ, 222, 1

Landolt A.U., 1983, AJ., 88, 439Michard R., 1985, A\&AS, 59, 205

Michard R., 1985, A\&AS, 59, 205

Sanchez-Blazquez P., Forbes D.A., Strader J., Brodie J., Proctor R., 2007, MNRAS, 377, 759 